\title{
New Results on the Equivalence of $K$-Functionals and Modulus of Continuity of Functions Defined on the Sobolev Space Constructed by the Generalized Jacobi-Dunkl Operator
}

\author{
Ali El Mfadel $(\mathbb{D}$, Said Melliani $\mathbb{D}$, and M'hamed Elomari \\ Laboratory of Applied Mathematics and Scientific Computing, Sultan Moulay Slimane University, Beni Mellal, Morocco \\ Correspondence should be addressed to Ali El Mfadel; elmfadelali@gmail.com
}

Received 9 July 2021; Accepted 20 December 2021; Published 20 January 2022

Academic Editor: Mohammad Mirzazadeh

Copyright (C) 2022 Ali El Mfadel et al. This is an open access article distributed under the Creative Commons Attribution License, which permits unrestricted use, distribution, and reproduction in any medium, provided the original work is properly cited.

In this paper, we establish some new generalized results on the equivalence of $K$-functionals and modulus of continuity of functions defined on the Sobolev space $L_{\alpha, \beta}^{2}(\mathbb{R})$, by using the harmonic analysis related to the Jacobi-Dunkl operator $\Delta_{\alpha, \beta}$, where $\alpha \geq \beta \geq-1 / 2$ and $\alpha \neq-1 / 2$.

\section{Introduction}

The modulus of continuity plays a fundamental role in the approximation theory of functions. For a given positive real number $r$ and a positive integer $m$, the classical modulus of continuity of a function $f \in L^{2}(\mathbb{R})$ is defined by

$$
\omega_{m}(f, r)=\sup _{0<h \leq r}\left\|D_{h}^{m} f\right\|_{L^{2}},
$$

where $D_{h}^{m} f=\left(\mathscr{T}_{h}-I\right)^{m} f$, such that $I$ is the unit operator in $L^{2}(\mathbb{R})$ and $T_{h}$ represents the usual translation operator given by $\mathscr{T}_{h} f(x)=f(x+h)$.

The classical $K$-functional, introduced by Peetre in [1], is defined by

$$
K_{p}(f, r)=\inf \left\{\|f-g\|_{L^{2}}+r\left\|D^{p} g\right\|_{L^{2}} ; g \in \mathscr{W}_{2}^{p}\right\},
$$

where $\mathscr{W}_{2}^{p}$ is the Sobolev space constructed by the operator $D=d / d x$ and

$$
\mathscr{W}_{2}^{p}:=\left\{f \in L^{2}(\mathbb{R}): D^{i} f \in L^{2}(\mathbb{R}), i=1, \cdots . ., p\right\} .
$$

A remarkable result of the approximation theory of functions on $\mathbb{R}$ which establishes the equivalence between the modulus of continuity and the $K$-functional can be formulated as follows:

Theorem 1 (see [2]). There exist two positive real constants $C_{1}$ and $C_{2}$ such that for any function $f \in L^{2}(\mathbb{R})$ and $r>0$, we have

$$
C_{1} \omega_{p}(f, r) \leq K_{p}\left(f, r^{p}\right) \leq C_{2} \omega_{p}(f, r) .
$$

The translation operator $f(x) \mapsto f(x+y)$ is used for the construction of modulus of continuity and smoothness which are the fundamental elements in the classical theory of the approximation of functions on $\mathbb{R}$ (see [3-5]).

The equivalence between the modulus of continuity and the $K$-functionals has been established in [6]. Various generalized continuity moduli are studied in $[7,8]$. A considerable attention has been devoted to finding generalizations of Theorem 1 (see, for example, [9-12]). This theorem was recently generalized in [13], for the Jacobi transform in the space $L^{2}\left(\mathbb{R}^{+}, A_{\alpha, \beta}(x) d x\right)$ by using a generalized Jacobi translation operator. Many generalized moduli of smoothness are often more convenient than the usual ones for the study of the connection between the smoothness properties of a function and the best approximations of this function in weight functional spaces (see, for example, [14]). 
Motivated by the results mentioned above, in this paper, we establish an analogue result of Theorem 1 by using the harmonic analysis associated with the Jacobi-Dunkl operator. The usual translation operators are replaced by generalized Jacobi-Dunkl translation operators in our new result.

Our paper is organized as follows: Section 2 gives some basic definitions, notations, lemmas, and theorems as preliminaries. Our main result is given in Section 3 followed by conclusion and future works in Section 4 .

\section{Preliminaries}

In this section, we give some notations, definitions, and results of the harmonic analysis related to the JacobiDunkl operator $\Lambda_{\alpha, \beta}$ defined by

$$
\begin{aligned}
\Lambda_{\alpha, \beta} f(x):= & \frac{d}{d x} f(x)+[(2 \alpha+1) \operatorname{coth} x+(2 \beta+1) \tanh x] \\
& \times \frac{f(x)-f(-x)}{2},
\end{aligned}
$$

where $\alpha \geq \beta \geq-1 / 2$ and $\alpha \neq-1 / 2$. We cite here, as briefly as possible, only those properties actually required for the discussion; for more details, we refer the reader to $[15,16]$.

The Jacobi-Dunkl Laplacian operator defined on $\mathbb{R}$ is given by

$$
\Delta_{\alpha, \beta} f(x):=\Lambda_{\alpha, \beta}^{2} f(x)
$$

which is equivalent to

$$
\Delta_{\alpha, \beta} f(x)=\mathscr{L}_{\alpha, \beta} f(x)+\frac{d}{d x}\left(\frac{\Lambda_{\alpha, \beta}^{\prime}}{\Lambda_{\alpha, \beta}}\right) f(x)\left(\frac{f(x)-f(-x)}{2}\right),
$$

where $\mathscr{L}_{\alpha, \beta}$ is the Jacobi operator defined by

$$
\mathscr{L}_{\alpha, \beta} f(x)=\frac{d^{2}}{d x^{2}} f(x)+[(2 \alpha+1) \operatorname{coth} x+(2 \beta+1) \tanh x] \frac{d}{d x} f(x) .
$$

In the rest of the paper, we denote the following:

(i) $\mathscr{D}(\mathbb{R})$ : the space of indefinitely differentiable functions on $\mathbb{R}$ with compact support

(ii) $\mathscr{D}_{p}(\mathbb{R})$ : the space of indefinitely differentiable pair functions on $\mathbb{R}$ with compact support

(iii) $\mathcal{S}(\mathbb{R})$ : the usual Schwartz space of indefinitely differentiable functions rapidly decreasing on $\mathbb{R}$, as well as their derivatives of all orders, provided with the topology of the following seminorms

$$
\begin{aligned}
L_{m, n}(f) & =\sup _{x \in \mathbb{R}, 0 \leq k \leq n}\left\{\left|\left(1+x^{2}\right)^{m}\right| \frac{d^{k}}{d x^{k}} f(x) \mid\right\}<+\infty, \\
\mathcal{S}^{1}(\mathbb{R}) & =\left\{(\cosh x)^{-2 \rho} f ; f \in \mathcal{S}(\mathbb{R})\right\} .
\end{aligned}
$$

This space is provided with the topology of the following seminorms $L_{m, n}^{1},(m, n) \in \mathbb{N}^{2}$, where

$$
L_{m, n}^{1}(f)=\sup _{x \in \mathbb{R}, 0 \leq k \leq n}\left[(\cosh x)^{-2 \rho}\left(1+x^{2}\right)^{m}\left|\frac{d^{k}}{d x^{k}} f(x)\right|\right]<+\infty .
$$

(iv) $L_{\alpha, \beta}^{p}(\mathbb{R}), p \geq 1$, and the space of measurable functions $f$ on $\mathbb{R}$ such that

$$
\|f\|_{p, \alpha, \beta}= \begin{cases}\left(\int_{\mathbb{R}}|f(x)|^{p} \mathscr{A}_{\alpha, \beta}(x) d x\right)^{1 / p}<+\infty, & \text { if } 1 \leq p<+\infty \\ \operatorname{essup}_{x \in \mathbb{R}}\{|f(x)|\}<+\infty, & \text { if } p=+\infty .\end{cases}
$$

where

$$
\mathscr{A}_{\alpha, \beta}(x)=2^{2(\alpha+\beta+1)}(\sinh |x|)^{2 \alpha+1}(\cosh |x|)^{2 \beta+1} .
$$

(v) $\mathscr{W}_{2, \alpha, \beta}^{m}$ is the Sobolev space constructed by the generalized Laplacian Jacobi-Dunkl operator, i.e.,

$$
\mathscr{W}_{2, \alpha, \beta}^{m}=\left\{f \in L_{\alpha, \beta}^{2}(\mathbb{R}): \Delta_{\alpha, \beta}^{i} f \in L_{\alpha, \beta}^{2}(\mathbb{R}), i=1,2, \cdots, m\right\},
$$

where $\Delta_{\alpha, \beta}^{0} f=f ; \Delta_{\alpha, \beta}^{i} f=\Delta_{\alpha, \beta}\left(\Delta_{\alpha, \beta}^{i-1} f\right), i=1,2, \cdots, m$.

Let $f \in L_{\alpha, \beta}^{2}(\mathbb{R})$ and $r>0$.

(vi) The generalized modulus of continuity of the function $f$ at order $m$ is defined by the following formula:

$$
\omega_{m}(f, r)_{2, \alpha, \beta}=\sup _{0<h \leq r}\left\|A_{h}^{m} f\right\|_{2, \alpha, \beta}
$$

where

$$
A_{h}^{m} f=\left(\mathscr{T}_{h}+\mathscr{T}_{-h}-2 I\right)^{m} f, \quad m=1,2, \cdots,
$$

and $I$ is the unit operator of the space $L_{\alpha, \beta}^{2}(\mathbb{R})$. 
(vii) Gauss's hypergeometric function ${ }_{2} F_{1}(a, b ; c ; z)$ is defined by

$$
{ }_{2} F_{1}(a, b ; c ; z):=\sum_{l=0}^{+\infty} \frac{(a)_{l}(b)_{l}}{(c)_{l} l !} z^{l}, \quad|z|<1,
$$

where $a, b, c, z \in \mathbb{C}, c \notin \mathbb{Z}^{-}$, and $(a)_{l}$ is the Pochhammer symbol given by

$$
(a)_{l}:= \begin{cases}a(a+1) \cdots(a+l-1), & \text { if } l \in \mathbb{N}^{*}, \\ 1, & \text { if } l=0 .\end{cases}
$$

where $\mathbb{N}^{*}$ is the set of nonzero positive integers.

(viii) The Jacobi function $\varphi_{\mu}^{\alpha, \beta}$ is defined by

$$
\varphi_{\mu}^{\alpha, \beta}(x)={ }_{2} F_{1}\left(\frac{\rho+i \mu}{2}, \frac{\rho-i \mu}{2} ; \alpha+1 ;-(\sinh x)^{2}\right) .
$$

(ix) The generalized $K$-functional of a function $f$ is defined by

$$
\begin{aligned}
& K\left(f, r, L_{\alpha, \beta}^{2}(\mathbb{R}), \mathscr{W}_{2, \alpha, \beta}^{m}\right) \\
& =\inf \left\{\|f-g\|_{2, \alpha, \beta}+r\left\|\Delta_{\alpha, \beta}^{m} g\right\|_{2, \alpha, \beta}: g \in \mathscr{W}_{2, \alpha, \beta}^{m}\right\},
\end{aligned}
$$

where $\Delta_{\alpha, \beta}$ is the generalized Jacobi-Dunkl Laplacian operator defined by (6).

For the short form, we denote

$$
K_{m}(f, r)_{2, \alpha, \beta}=K\left(f, r, L_{\alpha, \beta}^{2}(\mathbb{R}), \mathscr{W}_{2, \alpha, \beta}^{m}\right)
$$

(x) The Jacobi-Dunkl kernel $\psi_{\lambda}^{\alpha, \beta} \in \mathscr{C}^{\infty}(\mathbb{R})$ is the unique solution of the following differential equation:

$$
\left\{\begin{array}{l}
\Lambda_{\alpha, \beta} u=i \lambda u, \quad \lambda \in \mathbb{C}, \\
u(0)=1
\end{array}\right.
$$

which is defined by

$$
\psi_{\lambda}^{\alpha, \beta}(x):= \begin{cases}\varphi_{\mu}^{\alpha, \beta}(x)-\frac{i}{\lambda} \frac{d}{d x} \varphi_{\mu}^{\alpha, \beta}(x), & \text { if } \lambda \in \mathbb{C}^{*}, \\ 1, & \text { if } \lambda=0,\end{cases}
$$

where $\lambda^{2}=\mu^{2}+\rho^{2}, \rho=\alpha+\beta+1$, and $\varphi_{\mu}^{\alpha, \beta}$ is the Jacobi function given by (18). (xi) The Jacobi-Dunkl transform of a function $f \in \mathscr{D}(\mathbb{R})$ is defined by

$$
\mathscr{F}_{\alpha, \beta}(f)(\lambda)=\int_{\mathbb{R}} f(x) \psi_{\lambda}^{\alpha, \beta}(x) \mathscr{A}_{\alpha, \beta}(x) d x .
$$

Property 2 (see [17]).

(1) If $f \in L_{\alpha, \beta}^{1}(\mathbb{R})$, then $\mathscr{F}_{\alpha, \beta}(f) \in L_{\sigma}^{\infty}(\mathbb{R})$ and we have

$$
\left\|\mathscr{F}_{\alpha, \beta}(f)\right\|_{\infty, \sigma} \leq\|f\|_{1, \alpha, \beta}
$$

(2) If $f \in \mathcal{S}^{1}(\mathbb{R})$, then

$$
\begin{gathered}
\mathscr{F}_{\alpha, \beta}\left(\Lambda_{\alpha, \beta} f\right)(x)=i x \mathscr{F}_{\alpha, \beta}(f)(x), \\
\mathscr{F}_{\alpha, \beta}\left(\Delta_{\alpha, \beta} f\right)(x)=-x^{2} \mathscr{F}_{\alpha, \beta}(f)(x) .
\end{gathered}
$$

Theorem 3 (see [17]).

(i) The Jacobi-Dunkl transform $\mathscr{F}_{\alpha, \beta}$ is a topological isomorphism between $\mathcal{S}^{1}(\mathbb{R})$ and $\mathcal{S}(\mathbb{R})$

(ii) The Jacobi-Dunkl transform $\mathscr{F}_{\alpha, \beta}$ is a topological isomorphism from $L_{\alpha, \beta}^{2}(\mathbb{R})$ to $L_{\sigma}^{2}(\mathbb{R})$, and we have

$$
\int_{\mathbb{R}}|f(x)|^{2} \mathscr{A}_{\alpha, \beta}(x) d x=\int_{\mathbb{R}}\left|\mathscr{F}_{\alpha, \beta}(f)(\lambda)\right|^{2} d \sigma(\lambda) .
$$

Theorem 4 (see [17]). Let $f \in L_{\alpha, \beta}^{1}(\mathbb{R})$ such that $\mathscr{F}_{\alpha, \beta}(f) \in$ $L_{\sigma}^{1}(\mathbb{R})$; then, we have

$$
f(x)=\int_{\mathbb{R}} \mathscr{F}_{\alpha, \beta}(f)(\lambda) \psi_{-\lambda}^{\alpha, \beta}(x) d \sigma(\lambda) .
$$

Definition 5. Let $f \in L_{\alpha, \beta}^{2}(\mathbb{R})$ and $v>0$; we define the function $P_{v}$ by

$$
\begin{aligned}
P_{v}(f)(x) & =\int_{-\sqrt{v^{2}+\rho^{2}}}^{\sqrt{v^{2}+\rho^{2}}} \mathscr{F}_{\alpha, \beta}(f)(\lambda) \psi_{-\lambda}^{\alpha, \beta}(x) d \sigma(\lambda) \\
& =\left(\mathscr{F}_{\alpha, \beta}\right)^{-1}\left(\mathscr{F}_{\alpha, \beta}(f)(\lambda) \chi_{v}(\lambda)\right),
\end{aligned}
$$

where $\chi_{v}(\lambda)$ is the characteristic function defined by

$$
\chi_{v}(\lambda)= \begin{cases}1, & \text { if }|\lambda| \leq \sqrt{v^{2}+\rho^{2}} \\ 0, & \text { if }|\lambda|>\sqrt{v^{2}+\rho^{2}}\end{cases}
$$

and $\left(\mathscr{F}_{\alpha, \beta}\right)^{-1}$ is the inverse Jacobi-Dunkl transform. 
Remark 6. We can easily prove that the function $P_{v}(f)$ is indefinitely differentiable and $P_{v}(f) \in \mathscr{W}_{2, \alpha, \beta}^{m}, \forall m \in \mathbb{N}$.

Lemma 7 (see [18]). Let $f \in \mathscr{W}_{2, \alpha, \beta}^{m}$ and $r>0$; then,

(1) $\mathscr{F}_{\alpha, \beta}\left(\Delta_{\alpha, \beta}^{m} f\right)(\xi)=(-1)^{m} \xi^{2 m} \mathscr{F}_{\alpha, \beta}(f)(\xi)$, for all $\xi \in \mathbb{R}$

(2) $\omega_{m}(f, r)_{2, \alpha, \beta} \leq 2^{m} r^{2 m}\left\|\Delta_{\alpha, \beta}^{m} f\right\|_{2, \alpha, \beta}$

Lemma 8 (see [18]). Let $f \in L_{\alpha, \beta}^{2}(\mathbb{R})$ and $h>0$; then,

$$
\left\|A_{h}^{m} f\right\|_{2, \alpha, \beta} \leq 10^{m}\|f\|_{2, \alpha, \beta} .
$$

\section{Main Result}

In this section, we give the main result of our paper which is a generalization of Theorem 1 .

Theorem 9. There exist two positive real constants $C_{1}$ and $C_{2}$ such that for any function $f \in L_{\alpha, \beta}^{2}(\mathbb{R})$ and positive constant $r>0$, we have

$$
c_{1} \omega_{m}(f, r)_{2, \alpha, \beta} \leq K_{m}\left(f, r^{2 m}\right)_{2, \alpha, \beta} \leq c_{2} \omega_{m}(f, r)_{2, \alpha, \beta} .
$$

In order to prove Theorem 9, we will need some preliminary results.

Proposition 10. If $f \in \mathscr{W}_{2, \alpha, \beta}^{m}$ and $h>0$, then

$$
\mathscr{F}_{\alpha, \beta}\left(A_{h}^{m} f\right)(\lambda)=2^{m}\left(\varphi_{\mu}^{(\alpha, \beta)}(h)-1\right)^{m} \mathscr{F}_{\alpha, \beta}(f)(\lambda) .
$$

Proof. By using (18) and (23), we obtain

$$
\begin{aligned}
\mathscr{F}_{\alpha, \beta}\left(A_{h} f\right)(\lambda) & =\mathscr{F}_{\alpha, \beta}\left(\mathscr{T}_{h} f+T_{-h} f-2 f\right)(\lambda), \\
& =\left(\psi_{\lambda}^{(\alpha, \beta)}(h)+\psi_{\lambda}^{(\alpha, \beta)}(-h)-2\right) \mathscr{F}_{\alpha, \beta}(f)(\lambda), \\
& =2\left(\varphi_{\mu}^{(\alpha, \beta)}(h)-1\right) \mathscr{F}_{\alpha, \beta}(f)(\lambda) .
\end{aligned}
$$

We use the proof by induction for $m$, and we obtain the result.

Proposition 11. Let $f \in \mathscr{W}_{2, \alpha, \beta}^{m}$ and $r>0$; then, we have

$$
\omega_{m}(f, r)_{2, \alpha, \beta} \leq 2^{m} r^{2 m}\left\|\Delta_{\alpha, \beta}^{m} f\right\|_{2, \alpha, \beta} .
$$

Proof. Suppose that $h \in] 0, r]$. By using Lemmas 7 and 8 , we have

$$
\begin{aligned}
\left\|A_{h}^{m} f\right\|_{2, \alpha, \beta}^{2} & =\left\|\mathscr{F}_{\alpha, \beta}\left(A_{h}^{m} f\right)\right\|_{2, \sigma}^{2}=\int_{\mathbb{R}}\left|\mathscr{F}_{\alpha, \beta}\left(A_{h}^{m} f\right)(\lambda)\right|^{2} d \sigma(\lambda), \\
& =2^{2 m} \int_{\mathbb{R}}\left|\varphi_{\mu}^{(\alpha, \beta)}(h)-1\right|^{2 m}\left|\mathscr{F}_{\alpha, \beta}(f)(\lambda)\right|^{2} d \sigma(\lambda), \\
& \leq 2^{2 m} h^{4 m} \int_{\mathbb{R}} \lambda^{4 m}\left|\mathscr{F}_{\alpha, \beta}(f)(\lambda)\right|^{2} d \sigma(\lambda), \\
& =2^{2 m} h^{4 m} \int_{\mathbb{R}}\left|(-1)^{m} \lambda^{2 m} \mathscr{F}_{\alpha, \beta}(f)(\lambda)\right|^{2} d \sigma(\lambda), \\
& =2^{2 m} h^{4 m} \int_{\mathbb{R}}\left|\mathscr{F}_{\alpha, \beta}\left(\Delta_{\alpha, \beta}^{m} f\right)(\lambda)\right|^{2} d \sigma(\lambda), \\
& =2^{2 m} h^{4 m}\left\|\mathscr{F}_{\alpha, \beta}\left(\Delta_{\alpha, \beta}^{m} f\right)\right\|_{2, \sigma}^{2},=2^{2 m} h^{4 m}\left\|\Delta_{\alpha, \beta}^{m} f\right\|_{2, \alpha, \beta}^{2}
\end{aligned}
$$

thus,

$$
\left\|A_{h}^{m} f\right\|_{2, \alpha, \beta} \leq 2^{m} h^{2 m}\left\|\Delta_{\alpha, \beta}^{m} f\right\|_{2, \alpha, \beta}, \leq 2^{m} r^{2 m}\left\|\Lambda_{\alpha, \beta}^{m} f\right\|_{2, \alpha, \beta} .
$$

By taking the supremum on $h \in] 0, r]$, we obtain the result. $\square$

Proposition 12. Let $f \in L_{\alpha, \beta}^{2}(\mathbb{R})$ and $v>0$; then, there exists a positive number $c_{3}$ such that

$$
\left\|f-P_{v}(f)\right\|_{2, \alpha, \beta} \leq c_{3}\left\|A_{1 / v}^{m} f\right\|_{2, \alpha, \beta} .
$$

Proof. We use the Plancherel formula (26); we have

$$
\begin{aligned}
& \left\|f-P_{v}(f)\right\|_{2, \alpha, \beta}=\left\|\mathscr{F}_{\alpha, \beta}\left(f-P_{v}(f)\right)\right\|_{2, \sigma} \\
& =\left\|\left(1-\chi_{v}(\lambda)\right) \mathscr{F}_{\alpha, \beta}(f)(\lambda)\right\|_{2, \sigma} \\
& =\left\|\frac{1-\chi_{v}(\lambda)}{\left(1-\varphi_{\mu}^{(\alpha, \beta)}(1 / v)\right)^{m}}\left(1-\varphi_{\mu}^{(\alpha, \beta)}\left(\frac{1}{v}\right)\right)^{m} \mathscr{F}_{\alpha, \beta}(f)(\lambda)\right\|_{2, \sigma} .
\end{aligned}
$$

We use $\lambda^{2}=\mu^{2}+\rho^{2}$; then, we have

$$
|\lambda|>\sqrt{v^{2}+\rho^{2}} \Leftrightarrow \lambda^{2}>v^{2}+\rho^{2}, \Leftrightarrow \mu^{2}>v^{2}, \Leftrightarrow|\mu|>v .
$$

There exists $c>0$ such that

$$
\left(1-\varphi_{\mu}^{(\alpha, \beta)}\left(\frac{1}{v}\right)\right) \geq c
$$


for $\mu \in \mathbb{R}$ such that $|\mu| \geq \nu$, and by using Lemma 8 , we have

$$
\begin{aligned}
\left\|f-P_{v}(f)\right\|_{2, \alpha, \beta} & \leq c^{-m}\left\|\left(1-\varphi_{\mu}^{(\alpha, \beta)}\left(\frac{1}{v}\right)\right)^{m} \mathscr{F}_{\alpha, \beta}(f)(\lambda)\right\|_{2, \sigma} \\
& =c^{-m} 2^{-m}\left\|\mathscr{F}_{\alpha, \beta}\left(A_{1 / v}^{m} f\right)(\lambda)\right\|_{2, \sigma} \\
& =c_{3}\left\|A_{1 / v}^{m}(f)\right\|_{2, \alpha, \beta} .
\end{aligned}
$$

Proposition 13. Let $f \in L_{\alpha, \beta}^{2}(\mathbb{R})$ and $v>0$.

There exists a positive number $c_{4}$ such that

$$
\left\|\Delta_{\alpha, \beta}^{m}\left(P_{v}(f)\right)\right\|_{2, \alpha, \beta} \leq c_{4} v^{2 m}\left\|A_{1 / v}^{m} f\right\|_{2, \alpha, \beta} .
$$

Proof. We use the Plancherel formula (26) and Lemma 7; we have

$$
\begin{aligned}
& \left\|\Delta_{\alpha, \beta}^{m}\left(P_{v}(f)\right)\right\|_{2, \alpha, \beta}=\left\|\mathscr{F}_{\alpha, \beta}\left(\Delta_{\alpha, \beta}^{m}\left(P_{v}(f)\right)\right)\right\|_{2, \sigma^{\prime}} \\
& =\left\|\lambda^{2 m} \chi_{v}(\lambda) \mathscr{F}_{\alpha, \beta}(f)(\lambda)\right\|_{2, \sigma^{\prime}} \\
& =\|\| \frac{\lambda^{2 m}}{\left(1-\varphi_{\mu}^{(\alpha, \beta)}(1 / v)\right)^{m}}\left(1-\varphi_{\mu}^{(\alpha, \beta)}\left(\frac{1}{v}\right)\right)^{m} \chi_{v}(\lambda) \mathscr{F}_{\alpha, \beta}(f)(\lambda) \|_{2, \sigma} .
\end{aligned}
$$

For $|\lambda|>\rho$, we obtain

$$
\begin{aligned}
\sup _{\lambda \in \mathbb{R},|\lambda|>\rho} \frac{\lambda^{2 m} \chi_{v}(\lambda)}{\left|1-\varphi_{\mu}^{(\alpha, \beta)}(1 / v)\right|^{m}} & =v^{2 m} \sup _{\lambda \in \mathbb{R},|\lambda|>\rho} \frac{(\lambda / v)^{2 m} \chi_{v}(\lambda)}{\left|1-\varphi_{\mu}^{(\alpha, \beta)}(1 / v)\right|^{m}}, \\
& =v^{2 m} \sup _{|\lambda| \leq \sqrt{v^{2}+\rho^{2}},|\lambda|>\rho} \frac{(\lambda / v)^{2 m}}{\left|1-\varphi_{\mu}^{(\alpha, \beta)}(1 / v)\right|^{m}} .
\end{aligned}
$$

Note that if $v$ is large enough, then

$$
\frac{(\lambda / v)^{2 m}}{\left|1-\varphi_{\mu}^{(\alpha, \beta)}(1 / v)\right|^{m}} \longrightarrow 4^{m}(\alpha+1)^{m}
$$

thus

$$
c_{4}=\sup _{\rho<|\lambda| \leq \sqrt{\nu^{2}+\rho^{2}}} \frac{(\lambda / \nu)^{2 m}}{\left|1-\varphi_{\mu}^{(\alpha, \beta)}(1 / \nu)\right|^{m}} .
$$

Finally, formula (42) is proven.
Remark 14. Let $f \in L_{\alpha, \beta}^{2}(\mathbb{R})$ and $v>0$. There exists a positive constant $c_{4}$ such that

$$
\left\|\Delta_{\alpha, \beta}^{m}\left(P_{v}(f)\right)\right\|_{2, \alpha, \beta} \leq c_{4} v^{2 m} \omega_{m}\left(f, \frac{1}{v}\right)_{2, \alpha, \beta} .
$$

Proof of Theorem 9.

(i) We start by giving the proof of the inequality:

$$
c_{1} \omega_{m}(f, r)_{2, \alpha, \beta} \leq K_{m}\left(f, r^{2 m}\right)_{2, \alpha, \beta} .
$$

For this purpose, let $h \in] 0, r]$ and $g \in \mathscr{W}_{2, \alpha, \beta}^{m}$.

By using Propositions 10 and 11, we have

$$
\begin{aligned}
\left\|A_{h}^{m} f\right\|_{2, \alpha, \beta}= & \left\|A_{h}^{m}(f-g+g)\right\|_{2, \alpha, \beta} \leq\left\|A_{h}^{m}(f-g)\right\|_{2, \alpha, \beta} \\
& +\left\|A_{h}^{m} g\right\|_{2, \alpha, \beta} \leq 10^{m}\|(f-g)\|_{2, \alpha, \beta} \\
& +2^{m} h^{2 m}\left\|\Delta_{\alpha, \beta}^{m} g\right\|_{2, \alpha, \beta} \leq 10^{m}\|(f-g)\|_{2, \alpha, \beta} \\
& +2^{m} r^{2 m}\left\|\Delta_{\alpha, \beta}^{m} g\right\|_{2, \alpha, \beta} \leq 10^{m} \\
& \cdot\left(\|(f-g)\|_{2, \alpha, \beta}+r^{2 m}\left\|\Delta_{\alpha, \beta}^{m} g\right\|_{2, \alpha, \beta}\right) .
\end{aligned}
$$

We calculate the supremum on $h \in] 0, r]$ and the infimum on $g \in \mathscr{W}_{2, \alpha, \beta}^{m}$; we deduce that

$$
c_{1} \omega_{m}(f, r)_{2, \alpha, \beta} \leq K_{m}\left(f, r^{2 m}\right)_{2, \alpha, \beta},
$$

where $c_{1}=10^{-m}$.

(ii) Now, we give the proof of the inequality:

$$
K_{m}\left(f, r^{2 m}\right)_{2, \alpha, \beta} \leq c_{2} \omega_{m}(f, r)_{2, \alpha, \beta} .
$$

Since $P_{v}(f) \in \mathscr{W}_{2, \alpha, \beta}^{m}$, then by using the definition of $K$ -functional, we obtain

$$
K_{m}\left(f, r^{2 m}\right)_{2, \alpha, \beta} \leq\left\|f-P_{v}(f)\right\|_{2, \alpha, \beta}+r^{2 m}\left\|\Delta_{\alpha, \beta}^{m}\left(P_{v}(f)\right)\right\|_{2, \alpha, \beta} .
$$

We use Remark 14; we have

$$
K_{m}\left(f, r^{2 m}\right)_{2, \alpha, \beta} \leq c_{3} \omega_{m}\left(f, \frac{1}{v}\right)_{2, \alpha, \beta}+c_{4}(r v)^{2 m} \omega_{m}\left(f, \frac{1}{v}\right)_{2, \alpha, \beta} .
$$

Since $v$ is a positive arbitrary value, then by setting $v=$ $1 / r$, we obtain 


$$
K_{m}\left(f, r^{2 m}\right)_{2, \alpha, \beta} \leq c_{2} \omega_{m}(f, r)_{2, \alpha, \beta},
$$

where $c_{2}=c_{3}+c_{4}$.

\section{Conclusion}

In this paper, we generalized and we proved some new results on the equivalence between the $K$-functionals and the modulus of continuity of functions defined on the Sobolev space by using the harmonic analysis related to the Jacobi-Dunkl operator.

\section{Data Availability}

The data used to support the findings of this study are available from the corresponding author upon request.

\section{Conflicts of Interest}

The authors declare that they have no conflicts of interest.

\section{References}

[1] J. Peetre, A Theory of Interpolation of Normed Spaces, Notes de Universidade de Brasilia, 1963.

[2] P. Butzer and R. Nessel, "Fourier analysis and approximation," Bulletin of the London Mathematical Society, vol. 1, 1973.

[3] M. S. Hashemi, E. Darvishi, and D. Baleanu, "A geometric approach for solving the density-dependent diffusion Nagumo equation," Advances in Difference Equations, vol. 2016, no. 1, 2016.

[4] M. A. Mourou and K. Trimeche, "Transmutation operators and Paley-Wiener theorem associated with a differentialdifference operator on the real line," Analysis and Applications, vol. 1, no. 1, pp. 43-70, 2003.

[5] M. K. Potapov, "Application of the operator of generalized translation in approximation theory," Vestnik Moskovskogo Universiteta, Seriya Matematika, Mekhanika, vol. 3, pp. 3848, 1998.

[6] F. Dai, "Some equivalence theorems with K-functionals," Journal of Approximation Theory, vol. 121, no. 1, pp. 143-157, 2003.

[7] E. S. Belkina and S. S. Platonov, "Equivalence of K-functionals and modulus of smoothness constructed by generalized Dunkl translations," Russian Mathematics, vol. 52, no. 8, pp. 1-11, 2008.

[8] J. Lofstrom and J. Peetre, "Approximation theorems connected with generalized translations," Mathematische Annalen, vol. 181, no. 4, pp. 255-268, 1969.

[9] Z. Ditzian and V. Totik, Moduli of smoothness, Springer, Berlin, 1987.

[10] A. El Mfadel and S. Melliani, "On the existence and uniqueness results for fuzzy linear and semilinear fractional evolution equations involving Caputo fractional derivative," Journal of Function Spaces, vol. 2021, Article ID 4099173, 7 pages, 2021.

[11] A. el Mfadel, S. Melliani, and M.'. Elomari, “A note on the stability analysis of fuzzy nonlinear fractional differential equations involving the Caputo fractional derivative," International Journal of Mathematics and Mathematical Sciences, vol. 2021, Article ID 7488524, 6 pages, 2021.
[12] R. Subaie and M. Mourou, "Equivalence of K-functionals and modulus of smoothness generated by a generalized Dunkl operator on the real line," Advances in Pure Mathematics, vol. 5, no. 6, pp. 367-376, 2015.

[13] M. S. Hashemi, M. Inc, B. Kilic, and A. AkgÃijl, "On solitons and invariant solutions of the magneto-electro-elastic circular rod," Waves in Random and Complex Media, vol. 26, no. 3, pp. 259-271, 2016.

[14] S. S. Platonov, "Generalized Bessel translations and certain problems of the theory of approximation of functions in the metrics of $\mathrm{L}_{(2, \alpha)}$, "Trudy Petrozavodskogo Gosudarstvennogo Universiteta, Seriya Matematika, vol. 7, pp. 70-82, 2000.

[15] N. Ben Salem and A. Ahmed Salem, "Convolution structure associated with the Jacobi-Dunkl operator on IR," The Ramanujan Journal, vol. 12, no. 3, pp. 359-378, 2006.

[16] M. M. Chaffar, N. Bettaibi, and A. Fitouhi, "Sobolev type spaces associated with the q-Rubin's operator," Le Matematiche, vol. 29, pp. 37-56, 2014.

[17] F. Chouchane, M. Mili, and K. Trimeche, "Positivity of the intertwining operator and harmonic analysis associated with the Jacobi-Dunkl operator on $\$\{\backslash$ mathbb $\mathrm{R}\} \$$," Analysis and Applications, vol. 1, no. 4, pp. 387-412, 2003.

[18] F. Chouchane, M. Mili, and K. Trimeche, "An $L^{p}$ version of Hardy's theorem for the Jacobi-Dunkl transform," Integral Transforms and Special Functions, vol. 15, no. 3, pp. 225237, 2004. 